\title{
Imaging of microwave field using ultracold atoms
}

\author{
Pascal Böhi, ${ }^{1}$ Max F. Riedel, ${ }^{1}$ Theodor W. Hänsch, ${ }^{1}$ and Philipp Treutlein ${ }^{1,2, a)}$ \\ ${ }^{1}$ Max-Planck-Institut für Quantenoptik and Fakultät für Physik, Ludwig-Maximilians-Universität, \\ 80799 München, Germany \\ ${ }^{2}$ Departement Physik, Universität Basel, CH-4056 Basel, Switzerland
}

(Received 9 April 2010; accepted 27 April 2010; published online 2 August 2010)

\begin{abstract}
We report a technique that uses clouds of ultracold atoms as sensitive, tunable, and noninvasive probes for microwave field imaging with micrometer spatial resolution. The microwave magnetic field components drive Rabi oscillations on atomic hyperfine transitions whose frequency can be tuned with a static magnetic field. Readout is accomplished using state-selective absorption imaging. Quantitative data extraction is simple and it is possible to reconstruct the distribution of microwave magnetic field amplitudes and phases. While we demonstrate two-dimensional imaging, an extension to three-dimensional imaging is straightforward. We use the method to determine the microwave near-field distribution around a coplanar waveguide integrated on an atom chip. (C) 2010 American Institute of Physics. [doi:10.1063/1.3470591]
\end{abstract}

Today, monolithic microwave integrated circuits (MMICs) are of great importance in science and technology. In particular, they constitute key building blocks of today's communication technology. ${ }^{1}$ MMICs also serve as main components of superconducting quantum processors. ${ }^{2}$ In our group, a simple MMIC structure has recently been used as a tool for quantum coherent manipulation of ultracold atoms on an atom chip. ${ }^{3}$

Function and failure analysis is of crucial importance for the design of MMICs as well as for simulation verification. ${ }^{4}$ External port measurements (e.g., using a network analyzer) offer only limited insight. The microwave (mw) near-field distribution on the device gives much more information, enabling specific improvement. Therefore, different methods have been developed to measure the spatial distribution of mw near-fields. ${ }^{5}$ These methods use diverse physical effects to measure the mw electric or magnetic field. They have in common that they scan the field distribution point-by-point.

Here we propose and experimentally demonstrate a highly parallel method that allows for non-invasive and complete (amplitudes and phases) imaging of the mw magnetic field distribution using clouds of ultracold atoms. ${ }^{6}$ In this method, the mw magnetic field drives resonant Rabi oscillations ${ }^{7}$ between two atomic hyperfine levels that can be detected using state-selective absorption imaging. ${ }^{8}$ The method offers micrometer spatial resolution and a mw magnetic field sensitivity in the $10^{-8} \mathrm{~T}$ range at frequencies of a few gigahertz. It is a frequency-domain, single-shot technique to measure a two-dimensional field distribution. The method can be extended to measure three-dimensional (3D) distributions slice by slice. Data extraction is simple, it offers a high dynamic range, and it is intrinsically calibrated since only well-known atomic properties enter in the analysis.

For the proof-of-principle experiment presented here, we use our atom chip setup, ${ }^{3}$ see Fig. 1. The chip has integrated mw coplanar waveguide (CPW) structures that were designed for quantum manipulation of ultracold atoms. ${ }^{3}$ To demonstrate our method, we analyze the mw magnetic field near this structure. In general, the device to be tested does not have to be integrated on an atom chip.

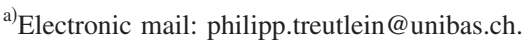

Our method works as follows. On the room-temperature atom chip, we prepare clouds of ${ }^{87} \mathrm{Rb}$ atoms at a temperature of $T \simeq 5 \mu \mathrm{K}$ using laser and evaporative cooling techniques. ${ }^{3,6}$ The magnetically trapped atoms are initially in the ground state hyperfine sublevel $\left|F, m_{F}\right\rangle=|1,-1\rangle$, see Fig. 1(a). The trap is moved close to the mw structure to be characterized, where it is switched off and the atoms are released to free fall. During a hold-off time $d t_{\text {ho }}$, the cloud drops due to gravity and expands due to its thermal velocity spread, filling the region to be imaged [Figs. 1(b) and 1(c)]. We maintain a homogeneous static magnetic field $\mathbf{B}_{0}$ of order $10^{-4} \mathrm{~T}$. It provides the quantization axis and splits the frequencies $\omega_{\gamma},(\gamma=-, \pi,+)$ of the three hyperfine transitions

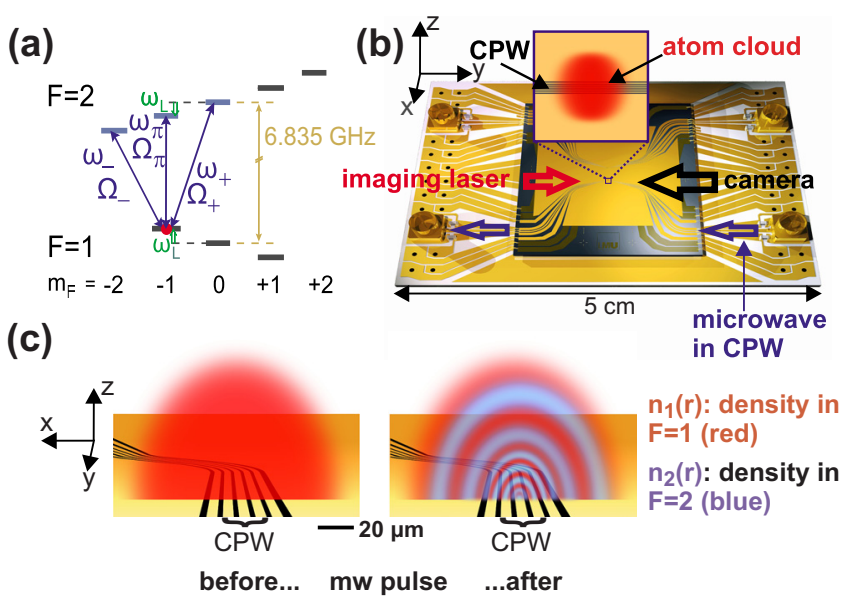

FIG. 1. (Color online) Schematic of the experiment. (a) Ground state hyperfine levels of ${ }^{87} \mathrm{Rb}$ atoms in a static magnetic field. Initially, the atoms are trapped in state $\left|F, m_{F}\right\rangle=|1,-1\rangle$. The three relevant transitions $|1,-1\rangle$ $\rightarrow\left|2, m_{2}\right\rangle\left(m_{2}=-2,-1,0\right)$ are indicated. The corresponding transition frequencies $\omega_{\gamma},(\gamma=-, \pi,+)$ are split by $\omega_{\mathrm{L}}$ due to the Zeeman effect. The Rabi frequencies $\Omega_{\gamma}$ are indicated. (b) The atom chip. Inset: Atom cloud near the CPW structure whose mw magnetic field is examined. The three inner wires constitute the CPW. (c) Experimental sequence. Left: The trap is switched off and the atom cloud expands. Right: A mw pulse is applied to the CPW, resonant with one of the transitions $\omega_{\gamma}$. Its magnetic field of amplitude $\mathbf{B}(\mathbf{r})$ drives Rabi oscillations with position-dependent $\Omega_{\gamma}(\mathbf{r})$ between $|1,-1\rangle$ and the corresponding state $\left|2, m_{2}\right\rangle$. The resulting atomic density distribution $n_{1}(\mathbf{r}) \quad\left(n_{2}(\mathbf{r})\right)$ in $F=1(F=2)$ is detected. From $n_{i}(\mathbf{r})$, we reconstruct $\Omega_{\gamma}(\mathbf{r})$. Several such measurements on the three transitions are combined to reconstruct $\mathbf{B}(\mathbf{r})$. 


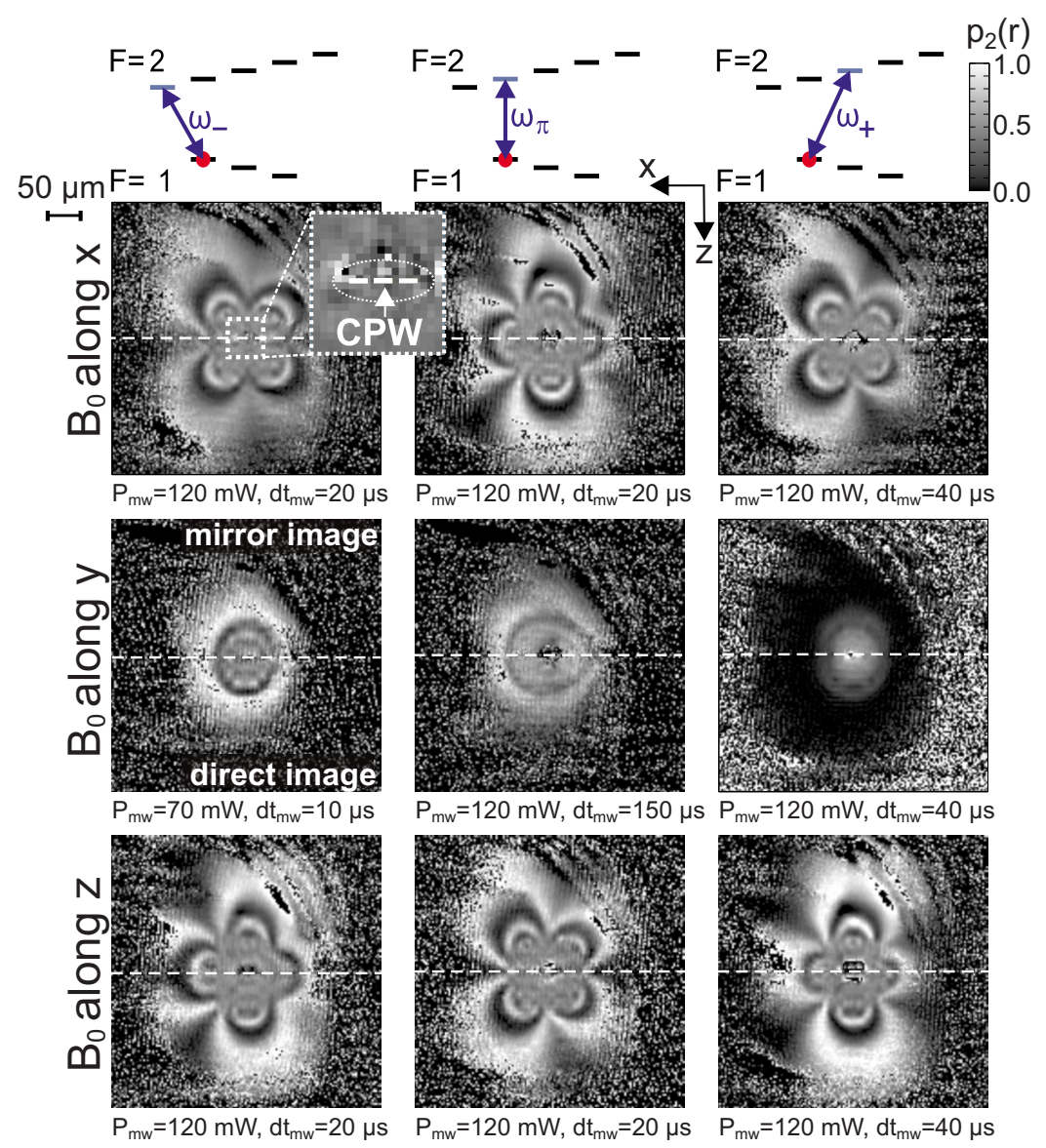

FIG. 2. (Color online) Imaging of mw magnetic field components near the CPW of Fig. 1. The images show the measured probability $p_{2}(\mathbf{r})$ to find an atom in $F=2$ after applying the mw pulse. Columns correspond to measurements on the three different transitions $\omega_{\gamma}$, rows to three different orientations of $\mathbf{B}_{0}$. The imaging beam is reflected from the chip surface at an angle of $2^{\circ}$. As a result, on each picture, a direct image and its reflection on the surface are visible. The dashed line separates the two. No atoms are visible in the very center of the image because the CPW structures distort the imaging beam. The mw power launched into the CPW, $P_{\mathrm{mw}}$, and the mw pulse duration $d t_{\mathrm{mw}}$ are indicated. $d t_{\mathrm{ho}}$ varies between 1 and $2 \mathrm{~ms}$. The noise in the image periphery corresponds to regions without atoms. Images are averaged over several experimental runs (15 to 130).

$|1,-1\rangle \rightarrow\left|2, m_{2}\right\rangle,\left(m_{2}=-2,-1,0\right)$ by $\omega_{\mathrm{L}}=\mu_{B} B_{0} / 2 \hbar$ due to the Zeeman effect [cf. Fig. 1(a)]. A mw signal on the MMIC is subsequently switched on for a duration $d t_{\mathrm{mw}}$ (typically some tens of microseconds). We select one of the transitions by setting the mw frequency $\omega=\omega_{\gamma}$. The mw magnetic field $\mathcal{B}(\mathbf{r}, t)=1 / 2\left[\mathbf{B}(\mathbf{r}) e^{-i \omega t}+\mathbf{B}^{*}(\mathbf{r}) e^{i \omega t}\right]$ couples to the atomic magnetic moment and drives Rabi oscillations of frequency $\Omega_{\gamma}(\mathbf{r})$ on the resonant transition. For the three transitions of interest,

$$
\begin{aligned}
& \Omega_{-}(\mathbf{r})=-\sqrt{3} \frac{\mu_{B}}{\hbar} B_{-}(\mathbf{r}) e^{-i \phi_{-}(\mathbf{r})}, \\
& \Omega_{\pi}(\mathbf{r})=-\sqrt{\frac{3}{4}} \frac{\mu_{B}}{\hbar} B_{\pi}(\mathbf{r}) e^{-i \phi_{\pi}(\mathbf{r})}, \\
& \Omega_{+}(\mathbf{r})=\sqrt{\frac{1}{2}} \frac{\mu_{B}}{\hbar} B_{+}(\mathbf{r}) e^{-i \phi_{+}(\mathbf{r})} .
\end{aligned}
$$

Here, $B_{\pi}$ and $\phi_{\pi}$ are the real-valued amplitude and phase of the component of $\mathbf{B}$ parallel to $\mathbf{B}_{0}$, and $B_{+}, \phi_{+}\left(B_{-}, \phi_{-}\right)$are the corresponding quantities for the right (left) handed circular polarization component in the plane perpendicular to $\mathbf{B}_{0}{ }^{9}$ After the mw pulse, a spatial pattern of atomic populations in $F=1$ and $F=2$ results, see Fig. 1(c). The probability to detect an atom in $F=2$ is

$$
p_{2}(\mathbf{r}) \equiv \frac{n_{2}(\mathbf{r})}{n_{1}(\mathbf{r})+n_{2}(\mathbf{r})}=\sin ^{2}\left[\frac{1}{2}\left|\Omega_{\gamma}(\mathbf{r})\right| d t_{\mathrm{mw}}\right] .
$$

Here, $n_{1}(\mathbf{r})\left(n_{2}(\mathbf{r})\right)$ is the density of atoms in $F=1 \quad(F=2)$, which can be measured using state-selective absorption imaging. ${ }^{8}$ From $p_{2}(\mathbf{r})$ we can reconstruct $\left|\Omega_{\gamma}(\mathbf{r})\right|$ and thus the spatial distribution of the resonant mw polarization component $B_{\gamma}(\mathbf{r})$, as shown below.

For a reconstruction of $\mathbf{B}(\mathbf{r})$, we measure $B_{\pi}(\mathbf{r}), B_{+}(\mathbf{r})$, and $B_{-}(\mathbf{r})$ with $\mathbf{B}_{0}$ pointing along the $x, y$, and $z$ axis. In each of the nine measurements (cf. Fig. 2), we tune $\omega$ into resonance with the desired transition $\omega_{\gamma}$. Alternatively, to test a device at a given frequency $\omega$, one can achieve resonance by Zeeman-tuning of $\omega_{\gamma}$ via $B_{0}$. The measurements allow a reconstruction of the amplitudes of all three Cartesian components of $\mathbf{B}$ as well as their relative phases. The measurements of $B_{\pi}$ for the three orientations of $\mathbf{B}_{0}$ directly yield the amplitudes $B_{x}, B_{y}$, and $B_{z}$. The relative phases can be reconstructed from the other measurements. ${ }^{9}$ It is also possible to measure the spatial dependence of the global phase of $\mathbf{B}$ using an interferometric method. ${ }^{9}$

Absorption imaging integrates over the direction of propagation of the imaging laser beam. Structures to be characterized should therefore have a characteristic length scale along the beam larger than the size of the atom cloud. The cloud size can be adjusted through the magnetic trap frequencies, $T$, and $d t_{\mathrm{ho}}$.

We experimentally demonstrate our method by measuring the mw magnetic field distribution near the $\mathrm{CPW}$ on our atom chip. The measurement is performed at a position where the CPW is translationally invariant along the imaging beam. An overview of the data is shown in Fig. 2. What appear in the images are essentially isopotential lines of the mw magnetic field components. Qualitative conclusions can be drawn directly by looking at the images-e.g., the left/ right asymmetry visible in Fig. 2 reveals that there is an asymmetry in the mw currents on the CPW wires. This con- 


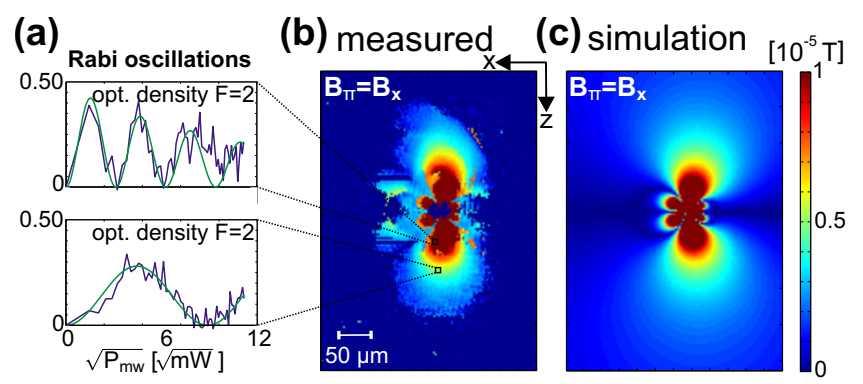

FIG. 3. (Color online) Measured mw magnetic field component $B_{x}(\mathbf{r})$ near the CPW and comparison with a simulation. The measurement is performed with $\omega=\omega_{\pi}$ and $\mathbf{B}_{0}$ along $x$, and the movie method (see text) is used to extract $B_{\pi}(\mathbf{r}) \equiv B_{x}(\mathbf{r})$. (a) Rabi oscillations at two exemplary pixels of the image, recorded by varying $P_{\mathrm{mw}}$ at fixed $d t_{\mathrm{mw}}=20 \mu \mathrm{s}$. The sinusoidal fits used to determine $\left|\Omega_{\pi}\right|$ and thus $B_{\pi} \equiv B_{x}$ as a function of $\sqrt{P_{\mathrm{mw}}}$ are shown. The observed decay of the oscillations is due to mw field gradients across the pixel. (b) Image of $B_{x}(\mathbf{r})$ at $P_{\mathrm{mw}}=120 \mathrm{~mW}$ as obtained from the data. (c) Corresponding quasistatic simulation of $B_{x}(\mathbf{r})$ (Ref. 3). We find best agreement with (b) if we allow for a $10 \%$ asymmetry between the currents on the two CPW ground wires and assume induced currents in the two wires next to the CPW grounds with an amplitude of $2 \%$ of the signal conductor current.

firms independent findings in our previous paper. ${ }^{3}$

It is possible to automatically extract $B_{\gamma}(\mathbf{r})$ from a single image. From $p_{2}(\mathbf{r})$ we can calculate $\left|\Omega_{\gamma}(\mathbf{r})\right|$ using Eq. (4) up to an offset of $n \pi / d t_{\mathrm{mw}}$, where $n$ is an integer. The offset for each point can be calculated by a ray-tracing method, where rays are sent from the image periphery (where $\left|\Omega_{\gamma}\right| d t_{\mathrm{mw}} \ll 1$ ) through the desired point to the center of the mw structure (where $\left|\Omega_{\gamma}\right|$ is maximal). $n$ is given by the sum of the number of minima and maxima of $p_{2}(\mathbf{r})$ encountered on the ray.

Alternatively, we take series of $k$ image frames, scanning either the mw power $P_{\mathrm{mw}}$ or $d t_{\mathrm{mw}}$ (the movie method). $k$ depends on the desired dynamic range but can be as low as 10. The time to record one frame is $14 \mathrm{~s}$ but could be reduced to $\leq 3 \mathrm{~s} .{ }^{10}$ For each image pixel, we thus obtain a sequence of $k$ datapoints showing Rabi oscillations, see Fig. 3(a). We fit a function $\propto \sin ^{2}\left[1 / 2\left|\Omega_{\gamma}\left(P_{\mathrm{mw}}\right)\right| d t_{\mathrm{mw}}\right]$ to the data, where $\left|\Omega_{\gamma}\left(P_{\mathrm{mw}}\right)\right|=a \sqrt{P_{\mathrm{mw}}}$ and $a$ is the fit parameter. From the fit, we determine $\left|\Omega_{\gamma}\right|$ and thus, via Eqs. (1)-(3), $B_{\gamma}$ at this pixel for a given $P_{\mathrm{mw}}$. As an example, Fig. 3(b) shows an image of the Cartesian mw field component $B_{x}(\mathbf{r})$ near our CPW reconstructed in this way. Figure 3(c) shows a corresponding simulation. Comparing data and simulation, we obtain information about the current distribution on the CPW.

The single-shot mw field sensitivity of our method is mainly determined by the interaction time $d t_{\mathrm{mw}}$. There is a trade-off between sensitivity and effective spatial resolution $s_{\text {eff. }}$ Longer $d t_{\mathrm{mw}}$ yields higher sensitivity but at the same time the image blurs due to the movement of the atoms. For our parameters (optical resolution $s_{\mathrm{opt}}=4 \mu \mathrm{m}, d t_{\mathrm{mw}}=80 \mu \mathrm{s}$, and $T=5 \mu \mathrm{K}$ ) and $d t_{\mathrm{ho}}=0$, we obtain $s_{\text {eff }}=8.2 \mu \mathrm{m}$ and a mw magnetic field sensitivity of $2 \times 10^{-8} \mathrm{~T}$.

In a variant of the presented method, trapped atoms could be used as a scanning mw field sensor. For trapped atoms, $d t_{\mathrm{mw}}$ can be much longer without increasing $s_{\text {eff }}$, thereby improving the field sensitivity. To record an image, the trap position has to be scanned from shot to shot of the experiment. ${ }^{9}$

Our method is frequency selective. Individual components of a multi-tone signal could be resolved. The transition frequencies $\omega_{\gamma}$ can be adjusted via $B_{0}$. For $B_{0}$ up to $0.5 \mathrm{~T}$, which is a realistic effort, e.g., for prototype testing, transition frequencies of $2.5-14 \mathrm{GHz}$ are accessible with ${ }^{87} \mathrm{Rb}$. ${ }^{9}$ Note that for $B_{0}>0.1 \mathrm{~T}$ we start entering the Paschen-Back regime, where the matrix elements of Eqs. (1)-(3) change and the theory has to be modified. Using other atomic species, different frequency ranges are accessible. Alternatively, a two-photon transition could be used for imaging, where two mw fields or a mw and a radio frequency field are applied to the atoms. The first field (frequency $\omega_{1}$ ) is applied externally with known spatial distribution, while the other field (frequency $\omega_{2}$ ) is to be imaged. Resonant Rabi oscillations occur for $\omega_{1}+\omega_{2}=\omega_{\gamma}$. It is also possible to image offresonant mw fields and other differential potentials using a Ramsey interferometry technique. ${ }^{9,11}$

Our technique can be extended to measure 3D distributions of $\mathbf{B}(\mathbf{r})$ slice by slice, either by using a gradient of $B_{0}$ such that only a slice of atoms is resonant with $\omega$, or by using a light sheet detection technique ${ }^{12}$ where slices perpendicular to the camera line of sight are imaged. Using similar techniques, it is also possible to shape the atomic cloud to prepare a thin sheet of atoms.

The method presented here seems promising for applications like prototype characterization. It allows for highly sensitive, parallel, high-resolution, and noninvasive imaging of the complete mw magnetic field distribution around an MMIC. Compact and portable systems for the preparation of ultracold atoms have been built, ${ }^{10}$ and key components of such systems are commercially available.

${ }^{1}$ I. D. Robertson and S. Lucyszyn, RFIC and MMIC Design and Technology, 1st ed. (The Institution of Electrical Engineers, London, 2001).

${ }^{2}$ L. DiCarlo, J. M. Chow, J. M. Gambetta, L. S. Bishop, B. R. Johnson, D. I. Schuster, J. Majer, A. Blais, L. Frunzio, S. M. Girvin, and R. J. Schoelkopf, Nature (London) 460, 240 (2009).

${ }^{3}$ P. Böhi, M. F. Riedel, J. Hoffrogge, J. Reichel, T. W. Hänsch, and P. Treutlein, Nat. Phys. 5, 592 (2009).

${ }^{4}$ C. Böhm, C. Roths, and E. Kubalek, IEEE MTT-S Int. Microwave Symp. Dig. 3, 1605 (1994).

${ }^{5}$ S. K. Dutta, C. P. Vlahacos, D. E. Steinhauer, A. S. Thanawala, B. J. Feenstra, F. C. Wellstood, S. M. Anlage, and H. S. Newman, Appl. Phys Lett. 74, 156 (1999); C. Böhm, F. Saurenbach, P. Taschner, C. Roths, and E. Kubalek, J. Phys. D: Appl. Phys. 26, 1801 (1993); Y. Gao and I. Wolff, IEEE Trans. Microwave Theory Tech. 46, 907 (1998); IEEE MTT-S Int. 3, 1159 (1995); G. David, P. Bussek, U. Auer, F. J. Tegude, and D. Jäger, Electron. Lett. 31, 2188 (1995); T. Dubois, S. Jarrix, A. Penarier, and P. Nouvel, IEEE Trans. Instrum. Meas. 57, 2398 (2008); T. P. Budka, S. D. Waclawik, and G. M. Rebeiz, IEEE Trans. Microwave Theory Tech. 44, 2174 (1996); R. C. Black, F. C. Wellstood, E. Dantsker, A. H. Miklich, D T. Nemeth, D. Koelle, F. Ludwig, and J. Clarke, Appl. Phys. Lett. 66, 1267 (1995)

${ }^{6}$ S. Chu, Nature (London) 416, 206 (2002).

${ }^{7}$ T. R. Gentile, B. J. Hughey, D. Kleppner, and T. W. Ducas, Phys. Rev. A 40, 5103 (1989).

${ }^{8}$ M. R. Matthews, D. S. Hall, D. S. Jin, J. R. Ensher, C. E. Wieman, E. A. Cornell, F. Dalfovo, C. Minniti, and S. Stringari, Phys. Rev. Lett. 81, 243 (1998).

${ }^{9}$ See supplementary material at http://dx.doi.org/10.1063/1.3470591 for further details on Rabi frequencies, reconstruction of the microwave field, sensitivity and spatial resolution, and tunability of transition frequencies $\omega_{-}, \omega_{\pi}$, and $\omega_{+}$.

${ }^{10}$ D. M. Farkas, K. M. Hudek, E. A. Salim, S. R. Segal, M. B. Squires, and D. Z. Anderson, Appl. Phys. Lett. 96, 093102 (2010).

${ }^{11}$ N. F. Ramsey, Molecular Beams (Clarendon, Oxford, 1956).

${ }^{12}$ R. Bücker, A. Perrin, S. Manz, T. Betz, C. Koller, T. Plisson, J. Rottmann, T. Schumm, and J. Schmiedmayer, New J. Phys. 11, 103039 (2009). 